\title{
Medida da potência térmica de refrigeração a água de uma bomba de vácuo difusora
}

\section{Water cooling thermal power measurement in a vacuum diffusion pump}

\author{
Luís Henrique Cardozo Amorin ${ }^{1}$; Paulo Rogério Catarini da Silva²; Jair Scarminio \\ Alexandre Urbano ${ }^{4}$.
}

\section{Resumo}

Bombas de vácuo difusoras são utilizadas na indústria e na ciência para produção de alto vácuo. Considerando que os sistemas de vácuo operam por horas e são refrigerados com água corrente, o consumo excessivo de água pode ser evitado se a refrigeração for realizada em circuito fechado. Contudo, para implementação de um destes circuitos é necessário se conhecer a potência térmica dissipada pela bomba, que pode ser obtida através do manual de operações do sistema, literatura técnica ou pela medida da própria potência dissipada pelo sistema, caso não se possa consultar o manual. Neste trabalho, a potência térmica de uma bomba difusora foi obtida medindo-se a vazão e a variação da temperatura da água, e calculada através da equação da variação de quantidade de calor em função do tempo. O valor encontrado foi de 935,6 W, que ao ser comparado com o manual de operações, está 397 $\mathrm{W}$ menor que a máxima potência e $35 \mathrm{~W}$ maior do que a mínima potência térmica da bomba. Com este procedimento é possível determinar com precisão, agilidade e facilidade, a potência térmica da bomba difusora ou de qualquer sistema que se pretende refrigerar a circuito fechado de água.

Palavras-chave: Bomba de vácuo difusora. Potência térmica. Circuito fechado de refrigeração.

\begin{abstract}
Diffusion vacuum pumps are used both in industry and in laboratory science for high vacuum production. For its operation they must be refrigerated, and it is done by circulating water in open circuit. Considering that, vacuum systems stays operating by hours, the water consumption may be avoided if the diffusion vacuum pumps refrigeration were done in closed circuit. However, it is necessary to know the diffusion vacuum pump thermal power (the heat transferred to circulate water by time units) to implement one of these and get in the refrigeration system dimension. In this paper the diffusion vacuum pump thermal power was obtained by measuring water flow and temperature variation and was calculated through the heat quantity variation equation time function. The thermal power value was $935,6 \mathrm{~W}$, that is $397 \mathrm{~W}$ smaller and $35 \mathrm{~W}$ bigger than, respectively, the maximum and minimum diffusion pump thermal power suggested by its operation manual. This procedure have been shown useful to precisely determine the diffusion pump thermal power or of any other system that needs to be refrigerated in water closed circuit.
\end{abstract}

Keywords: Diffusion Pump. Closed circuit refrigeration. Vacuum system.

\footnotetext{
${ }^{1}$ Aluno de mestrado do Departamento de Física, Universidade Estadual de Londrina; luis.amorin@gmail.com

2 Docente do Departamento de Física, Universidade Estadual de Londrina; presilva@uel.br

${ }^{3}$ Docente do Departamento de Física da Universidade Estadual de Londrina; scarmini@uel.br

${ }^{4}$ Docente do Departamento de Física da Universidade Estadual de Londrina; aurbano@uel.br
} 


\section{Introdução}

Bombas de vácuo difusoras são exemplos de equipamentos de apoio à pesquisa que necessitam serem refrigeradas à água para operarem adequadamente, tanto do ponto de vista técnico, para a produção de baixas pressões, como para manutenção da integridade física das mesmas (HARRIS, 1997), (ROTH, 1996), (OHRING, 1992). Para este tipo de bomba ser refrigerada em circuito fechado de água, porém, é necessário se dimensionar corretamente a potência do trocador de calor, que só pode ser feito conhecendo-se a potência térmica da bomba. Por potência térmica da bomba entenda-se o calor fornecido para a água de refrigeração por unidade de tempo.

Neste trabalho é apresentado um procedimento experimental para se obter a potência térmica de uma bomba difusora por meio da vazão e da variação da temperatura da água de refrigeração. A potência térmica é calculada pela equação da variação de quantidade de calor, absorvida pela água, em função do tempo. Esse processo é de uso geral, sendo aplicável ao cálculo da potência térmica de qualquer sistema que necessite ser refrigerado a circuito fechado de água.

\section{Fundamentos teóricos}

Uma bomba de vácuo difusora a óleo é um equipamento composto de um corpo cilíndrico, com duas aberturas, uma ligada à câmara a ser evacuada e outra por onde o gás bombeado é expelido (Figura 1). No seu interior é montada uma torre de difusão (castelo), que fica em contato com a base da bomba e que por sua vez é aquecida por uma resistência elétrica. Um óleo, de baixa pressão de vapor preenche a base da bomba e, ao ser aquecido, evapora por dentro do castelo sendo ejetado por difusores dispostos ao longo do mesmo. Bombas mecânicas são geralmente usadas para o pré-bombeamento da bomba difusora tendo a tarefa de diminuir a quantidade de moléculas oxidantes, ao mesmo tempo que gera uma diferença de pressão entre o interior e o exterior do castelo. Esta diferença de pressão é a responsável pela ejeção de moléculas de óleo pelos difusores que propositalmente as direciona para a base da bomba. As moléculas de óleo possuem elevado peso molecular e são ejetadas com altas velocidades, transferindo assim grande quantidade de momento às partículas de gás do entorno do castelo. As partículas do gás que são empurradas para a base da bomba difusora são sugadas pela bomba mecânica. Após sucessivas transferências de momento as moléculas de óleo perdem energia cinética e, para que o processo de bombeamento não cesse, estas devem ser coletadas e imobilizadas na parede interna da bomba, para que não contribuam com a elevação da pressão do sistema de vácuo. É por esse motivo que a parede do corpo da bomba deve ser refrigerada. Ao colidir com a parede fria as moléculas do óleo, que são de baixa pressão de vapor, condensam e com o sucessivo acúmulo escorrem de volta para o fundo da bomba, tornando o processo cíclico. A refrigeração da parede da bomba difusora é realizada externamente pela circulação de água em serpentinas enroladas na parede ou construindo-se bombas com paredes duplas.

Figura 1 - Esquema de uma bomba difusora em operação.

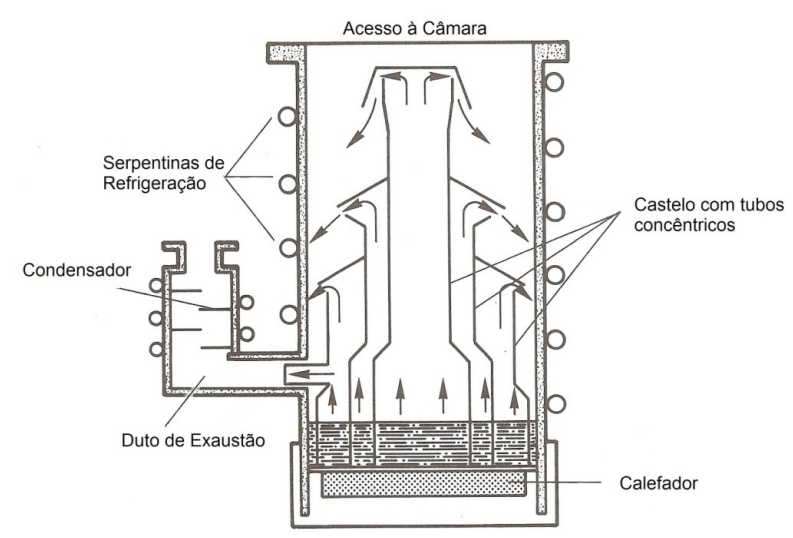

Fonte: Ohring, 1992. 


\section{Características técnicas de bombas difusoras}

De acordo com os dados dos manuais de operação de bombas difusoras, a velocidade de bombeamento (Vb), ou seja, a capacidade de bombeamento de gás em unidade de volume por unidade de tempo $(\mathrm{L} / \mathrm{s})$ de bombas de pequenos e médios portes (até 6000 $\mathrm{L} / \mathrm{s}$ ) é linearmente proporcional à potência elétrica consumida pela sua resistência de aquecimento do óleo. Por sua vez, a vazão de água requerida para refrigeração dessas bombas aumenta com a velocidade de bombeamento, logo, com sua potência elétrica (VARIAN DIFFUSION PUMPS, 2009), (EDWARDS VACUUM, 2009), (LEYBOLD VACUUM, 2009). Para bombas de vácuo difusoras com $\mathrm{Vb}$ de $160 \mathrm{~L} / \mathrm{s}$ a vazão de água recomendada é de $9 \mathrm{~mL} / \mathrm{s}$, enquanto para bombas com $\mathrm{Vb}$ de $6000 \mathrm{~L} / \mathrm{s}$ a vazão de água deve ser de $111 \mathrm{~mL} / \mathrm{s}$. É recomendado, nos manuais das bombas, que a temperatura da água na entrada esteja entre 15 e 26 ${ }^{\circ} \mathrm{C}$, e que a temperatura de saída não ultrapasse 48,5 ${ }^{\circ} \mathrm{C}$. Essas temperaturas são comuns para todos os modelos de bombas difusoras, uma vez que o óleo deve aquecer o suficiente para evaporar na base do castelo e condensar, sem congelar, na parede interna da mesma. O calor nas paredes do corpo da bomba é produzido pela condução térmica a partir da base aquecida, e internamente pelo vapor de óleo que condensa na mesma.

Quando uma bomba difusora é refrigerada com água corrente (circuito aberto) basta que as temperaturas citadas acima sejam monitoradas e controladas, pelo ajuste da vazão da água, para que a bomba opere com segurança. Entretanto, para a refrigeração da bomba em circuito fechado de água é necessário se conhecer a potência térmica a ser dissipada pela água refrigerante, para que seja usado um sistema de refrigeração que troque eficientemente o calor da água aquecida pela bomba. Uma estimativa da potência térmica de uma bomba pode ser obtida das especificações operacionais encontradas nos seus manuais de operação, avaliando-se os limites máximos e mínimos permitidos para a variação de temperatura sob a vazão mínima de água recomendada. Para a bomba investigada neste trabalho (VARIAN DIFFUSION PUMPS, 2009), cuja $\mathrm{Vb}=1200 \mathrm{~L} / \mathrm{s}$, utilizandose água como agente de refrigeração (densidade de $0,99 \mathrm{~g} / \mathrm{mL}$ ), sob a vazão mínima recomendada $(9,5 \mathrm{~mL} / \mathrm{s})$ e as variações de temperatura $(\Delta \mathrm{T})$ mínima e máxima permitidas, $22,9^{\circ} \mathrm{C}$ e $33,9^{\circ} \mathrm{C}$, as potências térmicas, mínima e máxima, transferidas para a água de refrigeração, serão respectivamente iguais a 900 e 1332 W. Estes valores são obtidos pela equação (5) discutida abaixo, usando para a densidade da água o valor de 0,99 g/mL. Contudo, como a potência térmica da bomba é constante, pois a sua potência elétrica também é constante, deve ser considerado que fatores externos, como a temperatura ambiente, podem contribuir para elevar a potência térmica recebida pela água que circula na mesma. Por essa razão, para se determinar com precisão a potência térmica da bomba, em qualquer que seja o ambiente, deve-se medi-la. A potência elétrica da bomba difusora estudada, segundo seu manual de operação, é de 1450 W.

\section{Potência térmica}

O valor da potência térmica da bomba difusora pode ser obtido com auxílio da conhecida equação da variação da quantidade de calor em função do tempo (NUSSENZVEIG, 1997), (HALLIDAY; RESNICK, 1987):

$$
\frac{d Q}{d t}=\frac{d m}{d t} c \Delta T+m c \frac{d \Delta T}{d t}
$$

Os termos desta equação são assim descritos: $\frac{d Q}{d t}$ ou $\mathrm{P}$, é a potência térmica de aquecimento $[\mathrm{J} / \mathrm{s}] ;: \frac{d m}{d t}$ é a vazão de massa $[\mathrm{g} / \mathrm{s}]$; c, é o calor específico $\left[\mathrm{J} / \mathrm{g}^{\circ} \mathrm{C}\right] ; \Delta \mathrm{T}$, é a variação da temperatura entre a entrada e a saída do sistema $\left[{ }^{\circ} \mathrm{C}\right]$; $\mathrm{m}$, é a massa $[\mathrm{g}]$ e $\frac{d \Delta T}{d t}\left[{ }^{\circ} \mathrm{C} / \mathrm{s}\right]$, corresponde à variação da 
temperatura da água entre a entrada e a saída em função do tempo.

Em regime estacionário, ou seja, com a bomba difusora plenamente aquecida e com equilíbrio entre as diferentes trocas de calor, o termo, $\frac{d \Delta T}{d t}$

é igual a zero. Assim a equação 1 se torna simplesmente,

$$
\mathrm{P}=\frac{\mathrm{dm}}{\mathrm{dt}} \mathrm{c} \Delta \mathrm{T}
$$

Sabendo que,

$$
\mathrm{dm}=\rho \mathrm{DV},
$$

e que,

$$
\varphi=\frac{\mathrm{dV}}{\mathrm{dt}}
$$

temos que,

$$
\mathrm{P}=\rho \varphi \mathrm{c} \Delta \mathrm{T}
$$

Na equação acima $\rho$ é a densidade da água [g/mL] e $\varphi$ a sua vazão volumétrica pela serpentina $[\mathrm{mL} / \mathrm{s}]$. Assim, a potência térmica de dissipação de calor por meio de água refrigerante será experimentalmente obtida medindo-se dois parâmetros, $\varphi$ e $\Delta \mathrm{T}$, em um sistema de refrigeração com água corrente.

\section{Metodologia experimental}

As potências térmicas calculadas neste trabalho referem-se à bomba difusora da marca Varian, modelo VHS 4, como mostrada na Figura 2 (VARIAN DIFFUSION PUMPS, 2009). A bomba foi fechada na parte superior (na conexão com a câmara a ser evacuada) e conectada em série, pelo duto de exaustão, com uma bomba mecânica rotativa de palhetas da marca Edwards modelo E2M8, para produção do pré-vácuo na difusora, e seu bombeamento, para exaustão de gás bombeado, quando em operação. A resistência de aquecimento do óleo da bomba difusora foi ligada (127 V) depois de a bomba ser evacuada à pressão de $10^{-2}$ mbar, medida por um sensor Piranni, com água corrente circulando na serpentina da mesma.
O regime estacionário de bombeamento foi experimentalmente observado medindo-se a temperatura da água de refrigeração na entrada e na saída da serpentina da bomba, por meio de um termopar, com precisão de $1{ }^{\circ} \mathrm{C}$, ligado a uma placa de aquisição de dados de um microcomputador. Na Figura 3 é mostrado o gráfico da temperatura da água versus o tempo (sob vazão de $21,59 \mathrm{~mL} / \mathrm{s}$ ), de onde se observa que a bomba atinge o regime estacionário após 20 minutos de funcionamento $\left(\frac{d \Delta T}{d t}=0\right)$. A temperatura ambiente de $20{ }^{\circ} \mathrm{C}$ manteve-se constante durante todo o tempo da análise.

Para o cálculo da potência térmica fornecida para a água, a vazão foi medida com auxílio de uma proveta com precisão de $0,05 \mathrm{~mL}$ e um cronômetro digital com precisão de $0,01 \mathrm{~s}$. A vazão foi variada em três níveis, $\varphi 1=10,66 \mathrm{~mL} / \mathrm{s}, \varphi 2=15,62 \mathrm{~mL} / \mathrm{s}$ e $\varphi 3=21,59 \mathrm{~mL} / \mathrm{s}$, sob a mesma temperatura de entrada de água, $\mathrm{T}_{\mathrm{e}}=17^{\circ} \mathrm{C}$. Cada valor de vazão foi obtido da média aritmética de dez medidas. Por sua vez a temperatura da água foi monitorada com termômetro de mercúrio com precisão de $0,05{ }^{\circ} \mathrm{C}$ e fundo de escala de $100{ }^{\circ} \mathrm{C}$. Dentro do intervalo de temperatura trabalhado considerou-se a densidade e o calor específico da água constantes, e respectivamente iguais a $\rho 0,99 \mathrm{~g} / \mathrm{mL}$ e $\mathrm{c}=4,18 \mathrm{~J} /$ $\mathrm{g}^{\circ} \mathrm{C}$.

Figura 2 - Bomba Difusora modelo VHS 4.

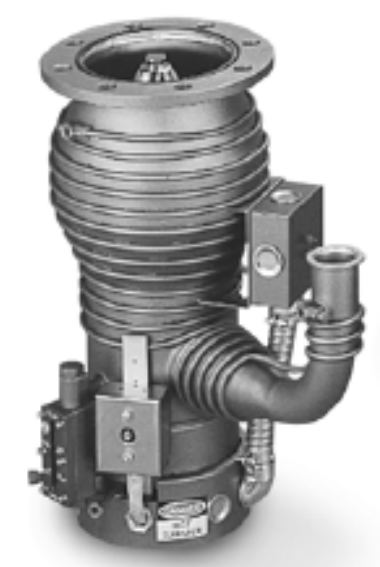

Fonte: LEYBOLD VACUUM, 2009 
Figura 3 - Gráfico da Variação da temperatura da água do sistema de refrigeração em função do tempo com respectivo ajuste

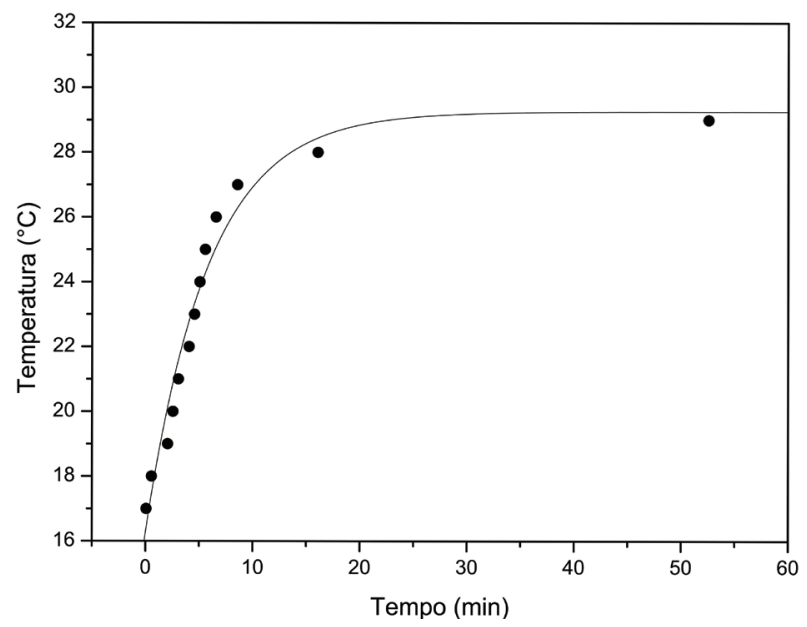

A potência elétrica do resistor de aquecimento da bomba difusora foi medida com um voltímetro e um amperímetro, conectados respectivamente em paralelo e em série com a resistência, em operação, e calculada utilizando-se a expressão $\mathrm{P}=\mathrm{Vi}$, onde $\mathrm{V}$ é a tensão aplicada e i a corrente elétrica resultante.

\section{Resultados}

A potência elétrica do resistor de aquecimento foi de $1615 \mathrm{~W}$, uma vez que a corrente elétrica medida foi de $13 \mathrm{~A}$, resultante da aplicação de uma tensão de 127 V. Esta potência elétrica é transformada em potência térmica por efeito Joule, usada em sua maior parte para aquecer o óleo da bomba e o restante aquecerá o corpo metálico da bomba e o ambiente.

Para o cálculo da potência térmica recebida pela água de refrigeração da bomba usou-se a equação (5). Para isto foram medidas as variações da temperatura $(\Delta \mathrm{T})$ da água para três diferentes vazões, com a bomba operando em regime estacionário. Os valores obtidos são mostrados na Tabela 1.
Tabela 1 - Variação de temperatura da água de refrigeração da bomba de vácuo difusora Varian VHS 4 operando em regime estacionário, em função da vazão da água para $T_{\mathrm{e}}=17^{\circ} \mathrm{C}$.

\begin{tabular}{lc}
\hline$\varphi(\mathrm{mL} / \mathrm{s})$ & $\Delta \mathrm{T}\left({ }^{\circ} \mathrm{C}\right)$ \\
\hline 10,66 & 21,20 \\
15,62 & 14,50 \\
21,59 & 10,60 \\
\hline
\end{tabular}

A potência térmica média recebida pela água refrigerante $(\mathrm{P})$, ou seja, sua taxa absorção de calor, foi obtida pelo ajuste linear da curva da variação da temperatura versus o inverso da vazão ( $\Delta \mathrm{T}$ vs. $1 / \varphi)$, como mostrado na Figura 4 , cujo coeficiente angular é igual a $\frac{\mathrm{P}}{\mathrm{pc}}$,

conforme a equação (5). O coeficiente angular obtido pelo ajuste foi de $226,5 \mathrm{~mL}^{\circ} \mathrm{C} / \mathrm{s}$, que resulta numa potência térmica média de 935,5 W. A Figura 4 mostra que a potência térmica é uma propriedade intrínseca do sistema analisado, sendo independe da vazão e do tipo de fluido refrigerante utilizado no circuito de refrigeração.

Figura 4 - Gráfico de $\Delta \mathrm{T}$ vs. $1 / \varphi$ com respectivo ajuste linear para obtenção da potência térmica.

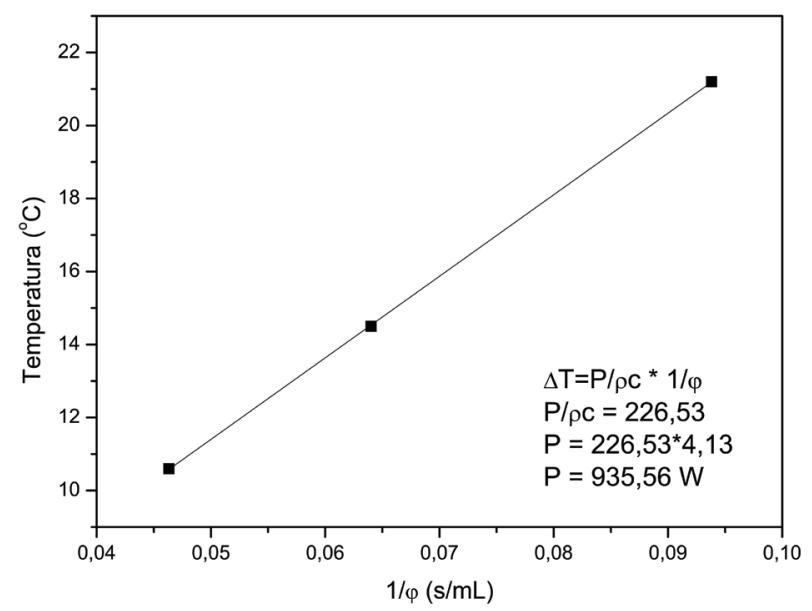




\section{Conclusões}

Obtivemos experimentalmente a potência térmica dissipada pela água de refrigeração de uma bomba de vácuo difusora, medindo-se a vazão da água corrente que circula através do circuito de refrigeração da bomba e sua variação de temperatura, entre a entrada no circuito e após sua saída. A potência térmica foi calculada por meio da equação de variação da quantidade de calor em função do tempo, com a bomba em regime estacionário para trocas de calor.

Conhecida a potência térmica dissipada pelo sistema de refrigeração em água corrente, ele pode ser substituído por outro com água em circuito fechado, já que este é o parâmetro fundamental para se dimensionar sistemas trocadores de calor. Esta é uma situação bastante comum em sistemas de vácuo com bombas difusoras, já que na sua maioria, a refrigeração é realizada com água corrente.

Para a bomba de vácuo analisada neste trabalho (Varian, modelo VHS 4), os dados técnicos da vazão da água e da variação de temperatura, indicam que para uma eficiente operação da bomba difusora, a potência térmica a ser dissipada pelo sistema de refrigeração a água corrente deve estar entre 900 e 1332 W. Como o valor encontrado experimentalmente para a potência de refrigeração foi de 935,6 W, pode-se afirmar que o sistema em questão está operando dentro dos limites de eficiência e segurança.

Para a bomba de vácuo analisada, a potência térmica gerada por efeito Joule no resistor de aquecimento foi de $1651 \mathrm{~W}$, distribuída como calor latente para vaporizar o óleo da bomba, como calor sensível para aquecer, por condução, o corpo e paredes metálicas da bomba e como calor radiante dissipado para o ambiente. Cerca de $57 \%$ deste calor $(935 \mathrm{~W})$ é retirado, através da refrigeração com água corrente, das paredes da bomba.

Com isso, a equação (5) pode ser também usada para se calcular diretamente a potência térmica de refrigeração de outros sistemas de refrigeração que operam em circuitos fechados, por exemplo, motores de combustão, lasers de alta potência etc.

\section{Referência}

DWARDS VACUUM. Diffstak vapor pumps. Disponível em: $\quad<$ http://www.edwardsvacuum.com/Products/List. aspx?r=41 >. Acesso em: 31 jul. 2009.

HALLIDAY, D.; RESNICK, R. Fundamentos de física, gravitação, ondas e termodinâmica. São Paulo: LTC, 1987.

HARRIS, S. N. Modern Vacuum Practice. Cambridge: McGraw-Hill, 1997.

LEYBOLD VACUUM. Oerlikon vacumm. Disponível em: $\quad<$ http://www.oerlikon.com/ecomaXL/index. php?site=OERLIKON_EN_segments_vacuum $>$. Acesso em: 31 jul. 2009.

NUSSENZVEIG, H. M. Curso de fisica básica, fluidos, oscilações, ondas e calor. São Paulo: Edgard Blücher, 1997.

OHRING, M. The materials science of thin films. London: Academic Press, 1992.

ROTH, A. Vacuum technology. Amsterdam: Elsevier, 1996.

VARIAN DIFFUSION PUMPS. VHS-4 Diffusion Pump Specifications. Disponível em: <http://www.varianinc. com>. Acesso em: 31 jul. 2009.

Recebido em 24 Outubro 2011- Received on October 24, 2011.

Aceito em 1 Dezembro, 2011 - Accepted on December 1, 2011. 